\title{
GENETIC ANALYSIS BY MEANS OF DIALLEL GRAPH*
}

\author{
S. JANA \\ Crop Science Department, University of Soskatchewan, Saskatoon, Conoda S7N OWO
}

Received 20.v.74

\begin{abstract}
SUMmary
A complete diallel cross was made among eight homozygous barley genotypes. Four of the parents were commercially grown cultivars and the remaining four consisted of isogenic derivatives of a California cultivar, Atlas. In a replicated experiment grown in two seasons, measurements were recorded on days to heading, days to maturity, plant height, grain yield and seed size. These data were then used to estimate variances $(V r)$ and covariances $(W r)$ of arrays in each season and a genetical interpretation of the relations between these statistics was sought. For days to heading, days to maturity and plant height, the $W r / V r$ graphs provided an evaluation of genetic relationship among the parental genotypes. As expected on the basis of their known genetic similarity, the isogenic lines of Atlas were close together on the $\mathrm{Wr} / \mathrm{Vr}$ graph. Contrary to the expectation, however, remarkable similarity among the commercially grown genotypes was indicated by the proximity of the array points representing them. The patterns of distribution of the array points were such that the eight parents could be classified into two wellseparated groups, one comprising the isogenic lines which carried most of the dominant alleles and the other comprising the cultivars carrying largely the recessive alleles. A seasonal difference in the genetic behaviour of the parents was also observed. Although some of the observed genetic differences between the two parental groups in one season disappeared in the following season, the positions of the array points were consistent in depicting substantial differences between the groups in both seasons. In most cases a graphical analysis was possible when a number of parents were removed from the diallel table. Non-random distribution of genes among the parents did not appear to be a common cause of disturbance in the relations between $\mathrm{Wr}$ and $V r$ even when the number of parents were very small. For two economically important characters, kernel size and grain yield, a graphical analysis was not possible, presumably because of the failure of a number of hypotheses underlying the analysis.
\end{abstract}

\section{INTRODUCtion}

THE regression graph of covariance $(W r)$ and variance $(V r)$ of arrays in diallel crosses provides a useful means of assessing genetic relationship among homozygous parents. As Jinks (1954) and Hayman (1954) have shown, if there are only two alleles at each locus, non-additive genetic variance is in the form of dominance only and the genes at the loci involved are distributed independently among the parents, then the linear regression of $W r$ on $V r$ has a unit slope. The effects of the failures of some of the assumptions on the diallel graph have been investigated and discussed by a number of authors (Hayman, 1957; Hill, 1964; Nassar, 1965; Mather, 1967; Coughtrey and Mather, 1970; etc.).

It appears that the condition of no non-allelic interaction (epistasis) is

* Supported by the Grant Number A6221 of the National Research Council of Canada.

$35 / 1-A$ 
particularly difficult to satisfy in many situations where diallel analysis is called for. Certain types of epistasis distort the $\mathrm{Wr} / \mathrm{Vr}$ graph in characteristic ways and thus permit their detection (Hayman, 1957; Mather, 1967; Coughtrey and Mather, 1970). Under favourable conditions, the departure from rectilinearity of the graph can be corrected by omitting the arrays corresponding to the disturbing and presumably epistatic parents. A useful genetic interpretation of the $W r / V r$ graph calculated from the remaining arrays can then be made on the basis of additive-dominance model. To further explore the applicability and usefulness of the above technique in understanding the genetic nature of parental variation, a diallel cross experiment involving eight non-randomly chosen homozygous genotypes of barley (Hordeum vulgare L.) was undertaken. The results of the experiment are reported and discussed in this paper.

\section{Materials AND Methods}

The materials consisted of a complete diallel cross among the following eight homozygous genotypes of barley:

\section{Group A}

Parent No.

1. Full-awned Atlas $(A A B B)$

2. Half-awned Atlas $(A A b b)$

3. Quarter-awned Atlas $(a a B B)$

4. Awnless Atlas (aabb)

\section{Group B}

Parent $\mathcal{N}$.

5. Bonanza

6. Galt

7. Gateway 63

8. Minnesota 5

The parents listed in group A are backcross-derived isogenic lines of the cultivar Atlas, developed by F. N. Briggs and G. W. Schaller at the University of California at Davis. A detailed account of the procedure used by Briggs and Schaller in developing the four lines was given by Qualset et al. (1965). The lines were isogenic except for two unlinked loci, designated as $A / a B / b$, which control lemma awn development. The genotypes of the lines with respect to the alleles at these two loci are given in the parentheses in the above list. In addition to the phenotypic differences for awn length, broadly classified as full-awned, half-awned, etc., the lines are known to differ for a number of quantitative characters (Qualset et al., 1965; Qualset, 1968; Schaller et al., 1972). Schaller et al. (1972) have suggested that the phenotypic variation among the isogenic lines may be ascribed to the chromosome segments measuring about five recombination units in length, linked with each of the marker genes.

Parents 5, 6 and 7 in group B are grown commercially on the Canadian Prairies. They are genetically diverse, morphologically distinguishable and differ significantly for a number of quantitative characters including days to heading, days to maturity, plant height and grain yield. Parent 8 in group B is a commercial cultivar grown in the state of Minnesota, but it is a poor grain yielder on the Prairies. Parents 5, 6 and 8 mature later and yield (seed) higher in Saskatoon then the isogenic lines of Atlas.

All possible $\mathrm{F}_{1}$ hybrids and their parents constituting a complete $8 \times 8$ diallel cross were grown in a summer nursery in Saskatoon in a randomised complete block design with two replications. Seed of each of the 64 entries in a replication was sown by hand in a three-row plot at a uniform distance 
of $45 \mathrm{~cm}$. The rows were $5 \mathrm{~m}$ long and $45 \mathrm{~cm}$ apart. Thus, except for a few ungerminated seeds, the spacing was $45 \mathrm{~cm}$ each way. This wide spacing was believed to minimise interplant competition. Measurements were recorded for 25 to 27 individual plants in each plot, excluding the end plants in each row. Data were collected on the following metrical characters:

(a) Days to heading: The number of days from sowing to the emergence of the first ear.

(b) Days to maturity: The number of days from sowing to yellowing of a plant to about 75 per cent.

(c) Plant height: Distance in centimetres from the soil surface to the apex of the tallest head, excluding the lemma awn.

(d) Kernel weight: Weight in grams of a random sample of 250 kernels $\times 4$.

(e) Grain yield: Kernel yield in grams after removing the awns.

The experiment was carried out for two years, 1971 and 1972, in the same investigation field but at different sites.

An analysis of variance of the randomised complete block design was performed for each trait on the plot mean basis, i.e. on the average of 25 to 27 plants for each entry in a replication. The data for each year were analysed separately.

The variance of each array $(V r)$ and the covariance of each array with the non-recurrent parents $(W r)$ were calculated for each replication from the diallel table of the means of reciprocal crosses. Tests of significance of the difference between arrays in $W r+V r$ and $W r-V r$ were performed by an analysis of variance. The array variances of the $W r+V r$ and $W r-V r$ values were compared with their respective variances for replicate blocks. If the ratio between mean squares for arrays and mean squares for replicate blocks was significant for $W r+V r$, but not for $W r-V r$, an adequate genetic basis was thought to exist to warrant the graphical analysis. However, for a further confirmation of the adequacy of a simple additive-dominance model uncomplicated by epistasis or the non-random distribution of genes among parents, a joint regression analysis of the $16 \mathrm{Wr}$ and $16 \mathrm{Vr}$ estimates was performed. Both methods of analysis have been described in detail by Mather and Jinks (1971).

If the regression coefficients for the two blocks were consistent and their joint regression coefficient was in agreement with the expected value of one, a new set of $V r$ and $W r$ values were calculated from the diallel table of the averages over replications and reciprocal $F_{1}$ families. These pairs of $(W r, V r)$ values represent the parental array points along the regression line in the geometric presentation of the diallel data. The linear regression of $W r$ on $V r$ was tested for significance $(\beta \neq 0)$ and for deviation from unity $(\beta \neq 1)$ by the usual $t$-tests.

When the joint regression of $W r$ on $V r$ for a trait deviated significantly from the value of one, one or more parents and their offspring in the arrays were omitted in turn until the expected rectilinear relation between $W r$ and $V r$ of the remaining arrays was achieved. Having removed the parents which caused most of the disturbances in the $W r / V r$ relationship, a genetic interpretation of the graph calculated from the remaining data was made on the basis of additive and dominance action of the independently distributed genes. The limitations and merits of this procedure of omitting epistatic members of a diallel set so that the remaining data conform to the 
simple genetic model are discussed by Jinks (1954, 1956), Allard (1956), Hayman (1957, 1963) and Johnson (1963).

\section{Analysis of means}

The means of the parental lines are given in table 1. A wide range of difference among the entries in the diallel cross was observed for all characters. An analysis of variance showed highly significant $(P=<0.001)$ differences among the entries in the $8 \times 8$ diallel cross, for days to heading, plant height, grain yield and seed size in both years. Entry differences in days to maturity were significant at $P=<0.001$ in 1971 and $\mathbf{P}=0 \cdot 010-0 \cdot 025$ in 1972 .

Relatively small differences were observed among the entries in the $4 \times 4$ subdiallel comprising the isogenic parents in group $\mathrm{A}$. When an

TABLE 1

The means of parents entering the diallel cross averaged over replicate blocks

\begin{tabular}{|c|c|c|c|c|c|c|c|c|c|}
\hline \multirow[b]{2}{*}{ Character } & \multirow[b]{2}{*}{ Year } & \multicolumn{8}{|c|}{ Mean of parents } \\
\hline & & 1 & 2 & 3 & 4 & 5 & 6 & 7 & 8 \\
\hline Days to heading & $\begin{array}{l}1971 \\
1972\end{array}$ & $\begin{array}{l}52 \cdot 70 \\
50 \cdot 78\end{array}$ & $\begin{array}{l}50 \cdot 77 \\
50 \cdot 20\end{array}$ & $\begin{array}{l}52 \cdot 26 \\
50 \cdot 96\end{array}$ & $\begin{array}{l}51 \cdot 21 \\
48 \cdot 61\end{array}$ & $\begin{array}{l}57 \cdot 65 \\
50 \cdot 90\end{array}$ & $\begin{array}{l}54 \cdot 20 \\
48 \cdot 72\end{array}$ & $\begin{array}{l}47.59 \\
41.46\end{array}$ & $\begin{array}{l}52 \cdot 00 \\
46 \cdot 62\end{array}$ \\
\hline Days to maturity & $\begin{array}{l}1971 \\
1972\end{array}$ & $\begin{array}{l}85 \cdot 61 \\
82.57\end{array}$ & $\begin{array}{l}84.05 \\
85.63\end{array}$ & & & $\begin{array}{l}95 \cdot 05 \\
87 \cdot 22\end{array}$ & & & $\begin{array}{l}99 \cdot 14 \\
88 \cdot 76\end{array}$ \\
\hline Plant height $(\mathrm{cm})$ & $\begin{array}{l}1971 \\
1972\end{array}$ & $\begin{array}{l}58 \cdot 26 \\
86 \cdot 63\end{array}$ & $\begin{array}{l}69 \cdot 71 \\
86 \cdot 64\end{array}$ & $\begin{array}{l}65 \cdot 62 \\
88 \cdot 77\end{array}$ & $\begin{array}{l}69 \cdot 98 \\
88 \cdot 29\end{array}$ & $\begin{array}{r}71 \cdot 19 \\
105 \cdot 12\end{array}$ & $\begin{array}{l}69 \cdot 65 \\
90 \cdot 01\end{array}$ & $\begin{array}{l}71 \cdot 59 \\
94.53\end{array}$ & $\begin{array}{l}78 \cdot 24 \\
97 \cdot 84\end{array}$ \\
\hline Kernel weight (gm) & $\begin{array}{l}1971 \\
1972\end{array}$ & $\begin{array}{l}32 \cdot 58 \\
44 \cdot 12\end{array}$ & $\begin{array}{l}29 \cdot 78 \\
41 \cdot 54\end{array}$ & $\begin{array}{l}25 \cdot 65 \\
39 \cdot 31\end{array}$ & $\begin{array}{l}27 \cdot 21 \\
36 \cdot 47\end{array}$ & $\begin{array}{l}35 \cdot 38 \\
43 \cdot 16\end{array}$ & $\begin{array}{l}39 \cdot 27 \\
45 \cdot 26\end{array}$ & $\begin{array}{l}30 \cdot 59 \\
42 \cdot 05\end{array}$ & $\begin{array}{l}40 \cdot 05 \\
45 \cdot 96\end{array}$ \\
\hline Grain yield (gm) & $\begin{array}{l}1971 \\
1972\end{array}$ & $\begin{array}{r}9 \cdot 27 \\
35 \cdot 41\end{array}$ & $\begin{array}{l}12.05 \\
28.98\end{array}$ & $\begin{array}{l}10 \cdot 73 \\
24 \cdot 19\end{array}$ & $\begin{array}{r}8 \cdot 76 \\
22 \cdot 33\end{array}$ & $\begin{array}{l}12 \cdot 90 \\
44 \cdot 37\end{array}$ & $\begin{array}{l}21 \cdot 14 \\
45 \cdot 14\end{array}$ & $\begin{array}{r}9 \cdot 80 \\
32 \cdot 11\end{array}$ & $\begin{array}{l}20 \cdot 87 \\
35 \cdot 96\end{array}$ \\
\hline
\end{tabular}

analysis of variance was performed for this group (subdiallel $A$ ), no difference was indicated among the entry means (table 2). On the other hand, considerably more variation was observed among the entries in the $4 \times 4$ subdiallel involving the parents in group $\mathbf{B}$ (subdiallel $B$ ). Separate analyses of variance for subdiallel $B$ indicated significant differences for most of the traits (table 2). Substantial differences were also observed between the two groups for most of the characters. The results are in general agreement with the expectation on the basis of the genetic closeness of isogenic parents and the diverse genetic origin of parental lines in group B. In the complete diallel, the bulk of genetic variation for a character was expected to be due to the differences among the parents in group $B$ and differences between the two groups.

\section{Analysis of $V r$ and $W r$}

(i) Days to heading

1971 data: Variances $(V r)$ and parent-progeny covariances $(W r)$ of individual arrays were estimated from the means of reciprocal families in each replication. An analysis of variance of $V r$ and $W r$ estimates showed 
significant array differences in the magnitude of $W r+V r(\mathrm{P}=0.001-0.002)$ and $W r-V r(\mathrm{P}=0.01-0.02)$. The heterogeneity of the $W r-V r$ values indicated the inadequacy of a simple additive-dominance model. The joint regression analysis of $W r$ and $V r$ showed consistency of regression coefficients over replications, but a poor linear fit, $b=0.374 \pm 0 \cdot 130$.

The estimates of mean days to heading over replications and reciprocal crosses were then used to obtain a set of eight $\mathrm{Vr}$ and $\mathrm{Wr}$ values. When the $W r / V r$ graph was calculated from these values, a poor linear regression was produced (fig. 1a). In an attempt to identify the disturbing parents, each parental array was omitted in turn and the remaining seven arrays were reanalysed. Array 5 had the largest $V r, W r$ and $W r-V r$ values. Yet, its removal did not yield a better graph. On the other hand, when

TABLE 2

Results of the analyses of variance for five metrical traits in two seasons

\begin{tabular}{|c|c|c|c|}
\hline \multirow[b]{2}{*}{ Character } & \multirow[b]{2}{*}{ Year } & \multicolumn{2}{|c|}{$\begin{array}{l}\text { Entry difference } \\
\text { (probability) }\end{array}$} \\
\hline & & Group A & Group B \\
\hline Days to heading & $\begin{array}{l}1971 \\
1972\end{array}$ & $\begin{array}{l}0 \cdot 10-0 \cdot 25 \\
0 \cdot 25-0 \cdot 50\end{array}$ & $\begin{array}{l}<0.001 \\
<0.001\end{array}$ \\
\hline Days to maturity & $\begin{array}{l}1971 \\
1972\end{array}$ & $\begin{array}{l}0 \cdot 10-0 \cdot 25 \\
0 \cdot 10-0 \cdot 25\end{array}$ & $\begin{array}{l}0.025-0 \cdot 05 \\
0.50-0 \cdot 75\end{array}$ \\
\hline Plant height & $\begin{array}{l}1971 \\
1972\end{array}$ & $\begin{array}{l}0 \cdot 05-0 \cdot 10 \\
0 \cdot 10-0 \cdot 25\end{array}$ & $\begin{array}{ll}0 \cdot 10 & -0.25 \\
0.01 & -0.025\end{array}$ \\
\hline Kernel size & $\begin{array}{l}1971 \\
1972\end{array}$ & $\begin{array}{l}0 \cdot 25-0 \cdot 50 \\
0 \cdot 50-0 \cdot 75\end{array}$ & $\begin{array}{l}0.005-0.01 \\
0.01-0.025\end{array}$ \\
\hline Grain yield & $\begin{array}{l}1971 \\
1972\end{array}$ & $\begin{array}{l}0 \cdot 50-0 \cdot 75 \\
0 \cdot 25-0 \cdot 50\end{array}$ & $\begin{array}{l}0 \cdot 005-0 \cdot 01 \\
0 \cdot 025-0 \cdot 05\end{array}$ \\
\hline
\end{tabular}

either array 1 or 7 was removed a substantial improvement of the graph was obtained, although the linear regression differed significantly from the expected slope of one. When both the arrays were eliminated from the diallel table and the remaining diallel set was reanalysed, the slope $(b=$ $0.802 \pm 0.236$ ) was significantly different from zero, but not from unity (fig. $1 \vec{b}$ ). Despite the improvement in the graphical relationship between $W r$ and $V r$ with the omission of arrays 1 and 7 , the analysis was of limited value as the size of the diallel set was much reduced $(6 \times 6)$ and a failure of one or more of the underlying assumptions was suggested by the heterogeneity of the $W r-V r$ values of the arrays. Non-random distribution of genes is likely to occur among the small number of parents which would tend to negate any gains made from the removal of the possible epistatic parents from the original diallel table.

The $W r / V r$ graph of the $4 \times 4$ subdiallel $B$ involving parents 5, 6, 7 and 8 produced a regression slope close to unity $(b=0.857 \pm 0.223)$, but it was not statistically significant $(\beta=0)$ due to the large standard error and the few degrees of freedom (fig. lc). However, the $W r-V r$ values appeared homogeneous over arrays by the $F$-test. The results strongly suggest that 
the parents in group A, either singly or collectively, caused upsets in the relationship between $W r$ and $V r$ in the complete $8 \times 8$ diallel population.

To pinpoint the offending parents, the four parents in group A were added one at a time to subdiallel $B$ and the analysis of the $5 \times 5$ diallel set was carried out as usual. It was found that the constancy of $\mathrm{Wr}-\mathrm{Vr}$ over arrays and the unit regression slope were lost only when either parent 1 or 3 was included in the analysis (fig. 2). In view of the small genetic

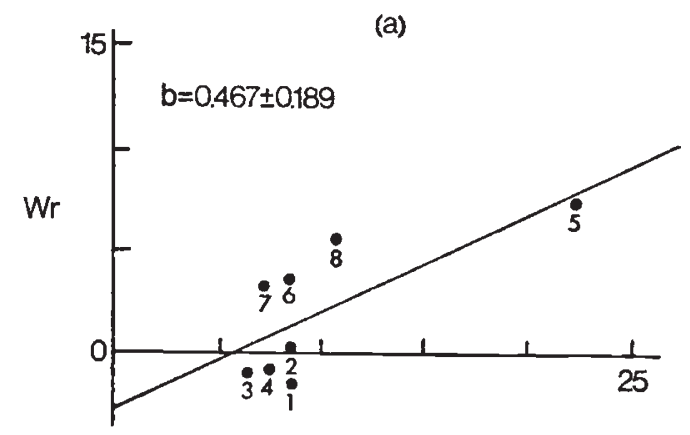

(b)

(c)

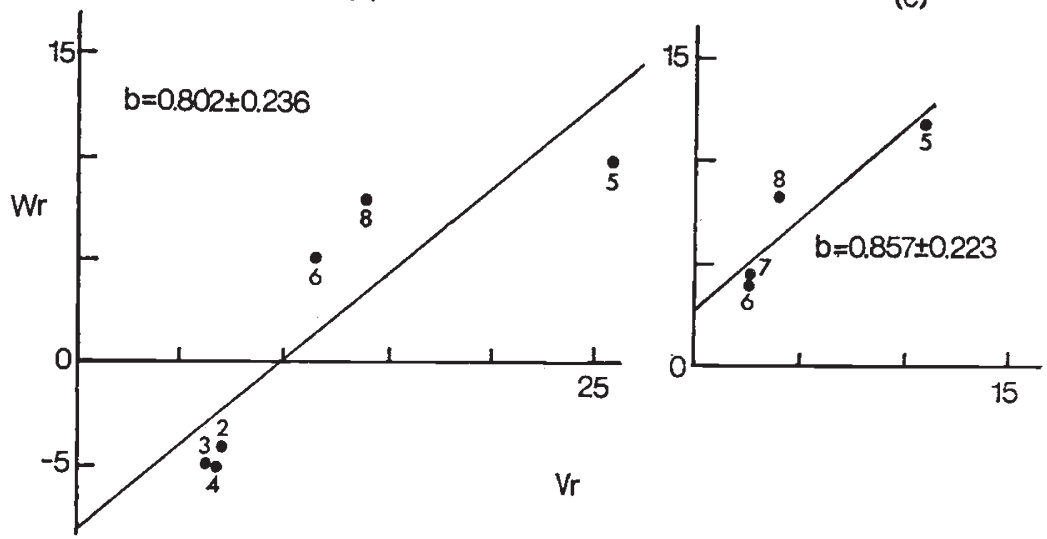

FIG. 1.-The regressions of $W r$ on $V r$ for days to heading in 1971; (a) all eight parents, $(b)$ parents 1 and 7 omitted, (c) group A omitted.

differences among the isogenic parents in group $\mathrm{A}$, the differential effects of these parents on the diallel graph are unexpected.

1972 data: An analysis of variance indicated significant $(\mathrm{P}=<0.001)$ array difference for $W r+V r$, but not for $W r-V r(\mathrm{P}=>0.75)$. However, the joint regression analysis revealed heterogeneity of regression over blocks and a joint regression, $b=0.505 \pm 0 \cdot 119$, which differed significantly from the value of one.

The $W r / V r$ graph calculated from the means over replications and reciprocal families also showed a poor regression (fig. $3 a$ ). Although small in magnitude, the difference between the regression coefficient $(b=$ $0 \cdot 742 \pm 0 \cdot 095)$ and the expected value of one was significant $(P=0 \cdot 02-0 \cdot 05)$. The relationship between $W r$ and $V r$ improved considerably when any one 
of parents 1, 4 and 5 was omitted, giving homogeneous differences between $W r$ and $V r$ over arrays and a unit regression slope. The removal of parental array 4 produced the most satisfactory regression graph (figure $3 b$ ). No further improvement of the graph was achieved by elimination of parents in pairs. The genetic differences among the parents in group $\mathrm{A}$ are small and attributable to the small chromosome segments marked by the alleles at the $A / a$ and $B / b$ loci. The effect on the $W r / V r$ graph of removing parents 1 or 4 was not expected to differ much from the effect of removing parent 2 or 3 . The differential consequences of the removal of single parents

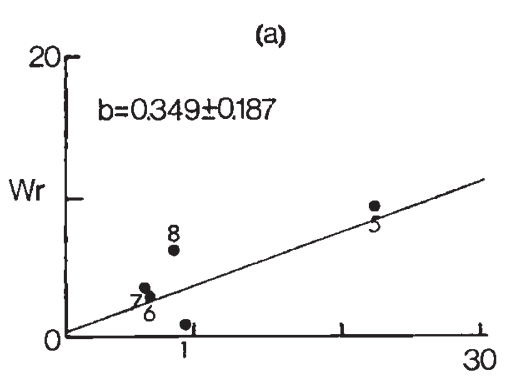

(C)

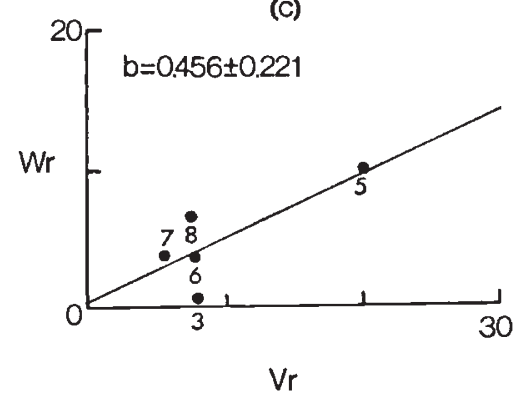

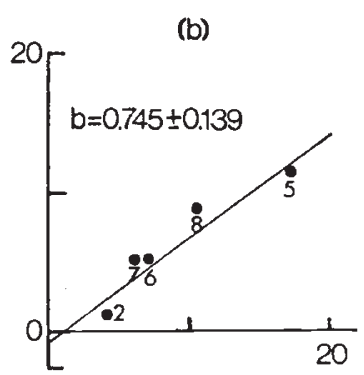

(d)

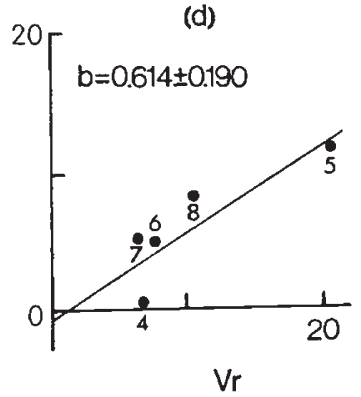

FIG. 2.-The regressions of $W r$ on $V r$ for days to heading in 1971 ; (a) parents 2,3 and 4 omitted, $(b)$ parents 1, 3 and 4 omitted, $(c)$ parents 1, 2 and 4 omitted, $(d)$ parents 1,2 and 3 omitted.

from the isogenic group must then be attributed to the differential effects of the chromosome segments marked by the $A / a$ and $B / b$ loci. If nonallelic interactions of some of the gene combinations in these segments were the major causes of disturbance, a graphical analysis excluding them would produce better results, provided non-random distribution of genes due to the drastic reduction in sample size did not cause serious upsets. The $W r / V r$ graphs of the subdiallel $B$ showed the expected rectilinear relation between $W r$ and $V r$ with unit slope (fig. $3 c$ ). The $W r-V r$ values were also homogeneous over arrays.

\section{(ii) Days to maturity}

1971 data: An analysis of variance showed that array differences in $W r+V r$ were significant $(\mathrm{P}=0.001)$, but the $W r-V r$ values over arrays were homogeneous $(P=>0 \cdot 75)$. The joint regression coefficient, $b=$ $1 \cdot 052 \pm 0.057$, differed from zero $(P=0.001)$ but not from unity $(P=0.2$ - 
0.4). Block differences in regression of $W r$ or $V r$ were not significant $(P=0.75)$. Thus both analyses were consistent in indicating that the simple additive-dominance model of independently distributed genes provided an adequate description of the diallel data on days to maturity.

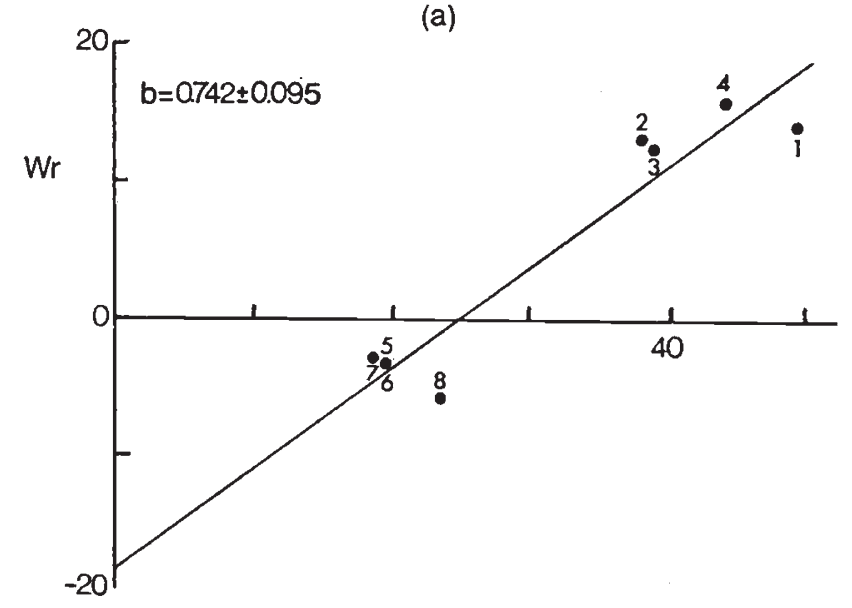

(b)

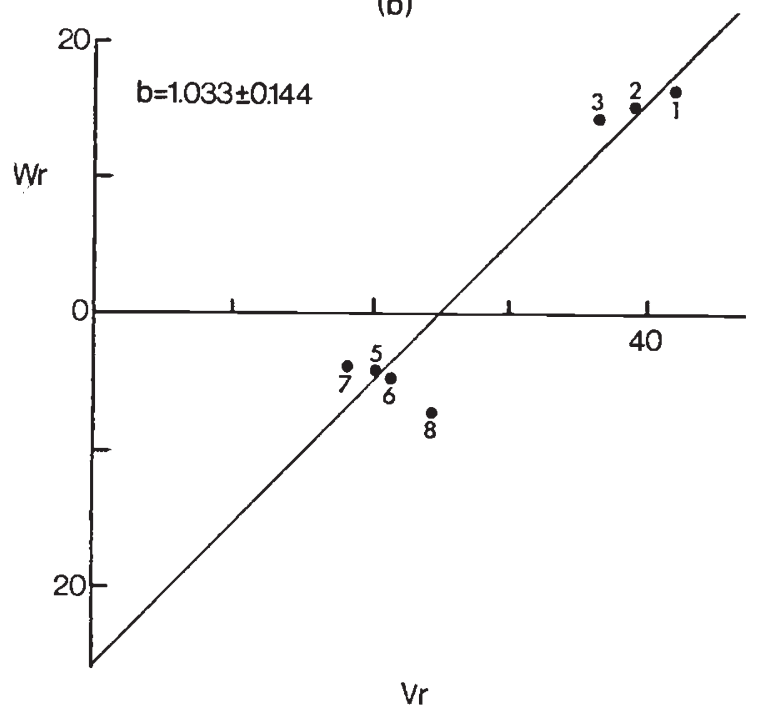

(c)

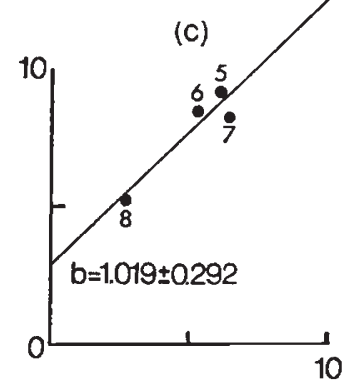

Fig. 3.-The regressions of $W r$ on $V r$ for days to heading in 1972; (a) all eight parents, $(b)$ parent 4 omitted, (c) group A omitted.

The $W r / V r$ graph, calculated from the average maturity time over blocks and reciprocals for each entry, confirms the rectilinear relationship between $W r$ and $V r$ (fig. $4 a$ ). The $W r$-intercept was close to the origin, indicating complete dominance, which was confirmed from the test of significance of $W r-V r$, corrected for environment. The positions of the $(V r, W r)$ points on the regression graph showed that most of the dominant 
alleles were present in the isogenic lines of Atlas and the four genotypes in group B had a preponderance of the recessive alleles. The proximity of the array points corresponding to the isogenic lines was a true reflection of their genotypic similarity. However, the remarkable closeness of the group B parents to one another near the point of intersection of the regression line and the limiting parabola is difficult to interpret without assuming a

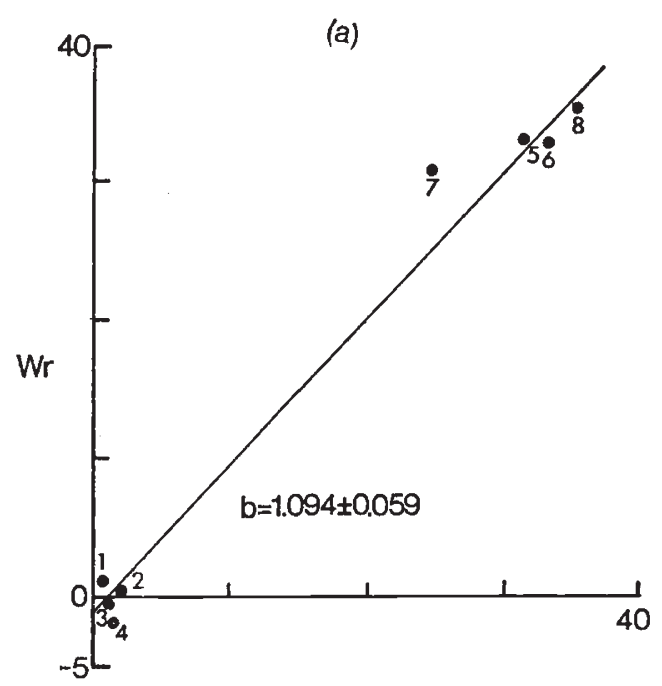

(c)

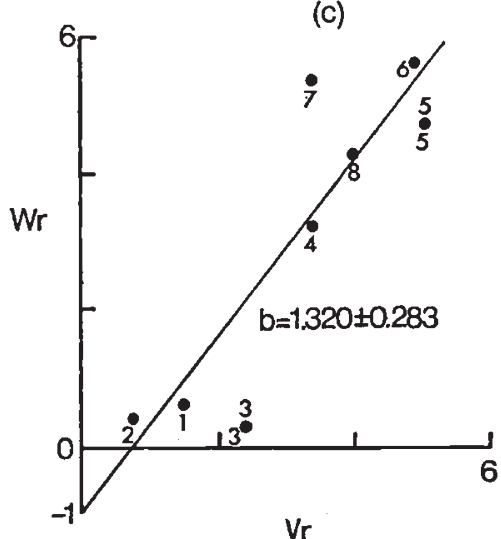

(b)

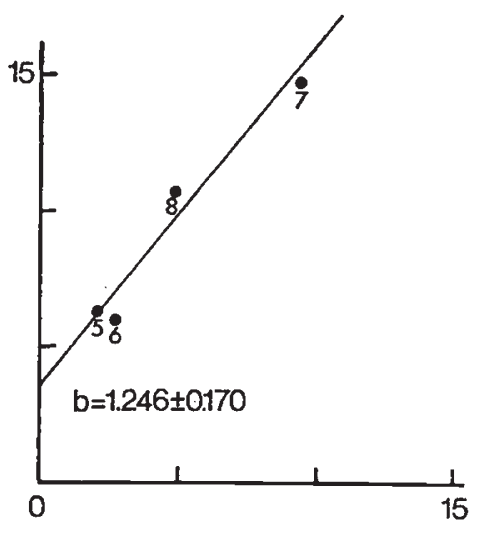

(d)

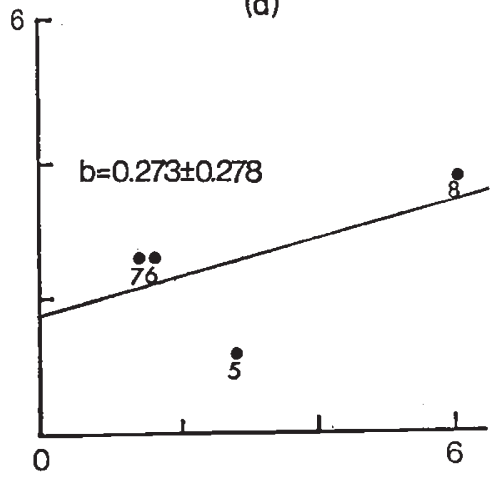

$\mathrm{Vr}$

Frg. 4.-The regressions of $W r$ on $V r$ for days to maturity; (a) all eight parents in 1971, (b) group A omitted in 1971, (c) all eight parents in 1972, (d) group A omitted in 1972.

comparable genetic similarity. From the striking discontinuity of the distribution of the array points it would appear that the pattern of genetic diversity is one of large intergroup and small intragroup differences.

Separate graphical analysis of subdiallel $A$ produced a regression coefficient differing neither from zero nor from unity. However, for subdiallel $B$, a rectilinear relation of $W r$ and $V r$ in a partially dominant genetic system was evident (fig. $4 b$ ).

1972 data: As in 1971, an analysis of variance of the estimates of $W r$ 
and $V r$ and a joint regression analysis were in agreement with regard to the additive-dominance mode of action of the genes, which were distributed randomly among the eight parents.

The $W r / V r$ graph showed a concentration of most of the dominant genes in the isogenic parents with the exception of parent 4, which appeared closer to the parents in group B than to the isogenic genotypes of its own group (fig. 4c). Although the pattern of distribution of dominant and recessive genes in the parents was, in general, comparable in both years, the intergroup difference was smaller and intragroup difference larger in 1972 than in the previous year. The $W r$-intercept was slightly below the origin and the average difference between $W r$ and $V r(\overline{W r}-\overline{V r})$ corrected for environment was significant $(P=<0.001)$ suggesting overdominance of the genes controlling days to maturity.

Separate graphical analysis of subdiallel $A$ provided no additional information. The slope of the regression line was close to unity, but did not differ significantly $(P=0 \cdot 05-0 \cdot 10)$ from zero. The regression graph for subdiallel $B$ revealed loss of rectilinearity (fig. $4 d$ ) which was not restored by the addition of any single isogenic line to the subset.

\section{(iii) Plant height}

1971 data: In an analysis of variance of the estimates of $W r$ and $V r$, the variance ratios between array differences and block differences were significant for both $W r+\operatorname{Vr}(\mathrm{P}=0.005-0.01)$ and $W r-V r(\mathrm{P}=0.25-0.05)$. The joint regression coefficient, $b=0.428 \pm 0.066$, was significantly different from both 0 and $1(P=<0.001)$. Furthermore, the regression coefficients for the two blocks (heterogenity of regression) were significantly different $(\mathrm{P}=<0.001)$. Thus both analyses were in agreement in showing the inadequacy of a simple additive-dominance model in the inheritance of plant height.

The departure from the expected unit linear relation between $W r$ and $\mathrm{Vr}$ was also evident from the regression graph (fig. 5a). Hayman (1957, 1963) recommended that, when such a departure occurred, it was desirable to separately eliminate the parents corresponding to the maximum and minimum $W r-V r$ values and examine the consequences of their removal on the $W r / V r$ graph. Although the removal of either parent 6 (minimum $W r-V r$ ) or parent 2 (maximum $W r-V r$ ) improved the graphical relationship, the best unit linear fit $(b=0.881 \pm 0.063)$ was obtained when parent 5 with an intermediate $W r-V r$ value was omitted. An improvement of the graph was also achieved by the joint removal of either parents 6 and 7 or parents 2 and 6 (fig. $5 b$ and c). Parents 7 and 6 had, respectively, the largest and second largest array variances and covariances. Considerably larger differences between $V r$ and $W r$ for these arrays than for the remaining six arrays were observed. Consequently their removal yielded homogeneous $W r-V r$ values over arrays and the best unit linear fit of the regression graph. However, the positions of the array points along the regression line did not show a separation of the parents into two well-defined groups as was observed in several other cases. The closeness of the array points corresponding to the isogenic parents in relation to their distance from the other members of the diallel set is not consistent with their genetic closeness. 
For instance, fig. $5 b$ and $c$ shows as much diversity between parents 2 and 4 , which are known to differ only in respect of the small chromosome segments marked by the $B / b$ locus as between parents 1 and 5 or 5 and 8 .

Separate analyses were then performed for subdiallels $A$ and $B$. Despite the closeness to unity $(b=0 \cdot 848 \pm 0.263)$, the slope of the regression line for subdiallel $A$ was not significantly different from zero $(P=0 \cdot 05-0 \cdot 10)$. The regression graph for the subdiallel $B$ also showed the absence of a rectilinear relation between $W r$ and $\operatorname{Vr}(b=0 \cdot 107 \pm 0 \cdot 230)$.
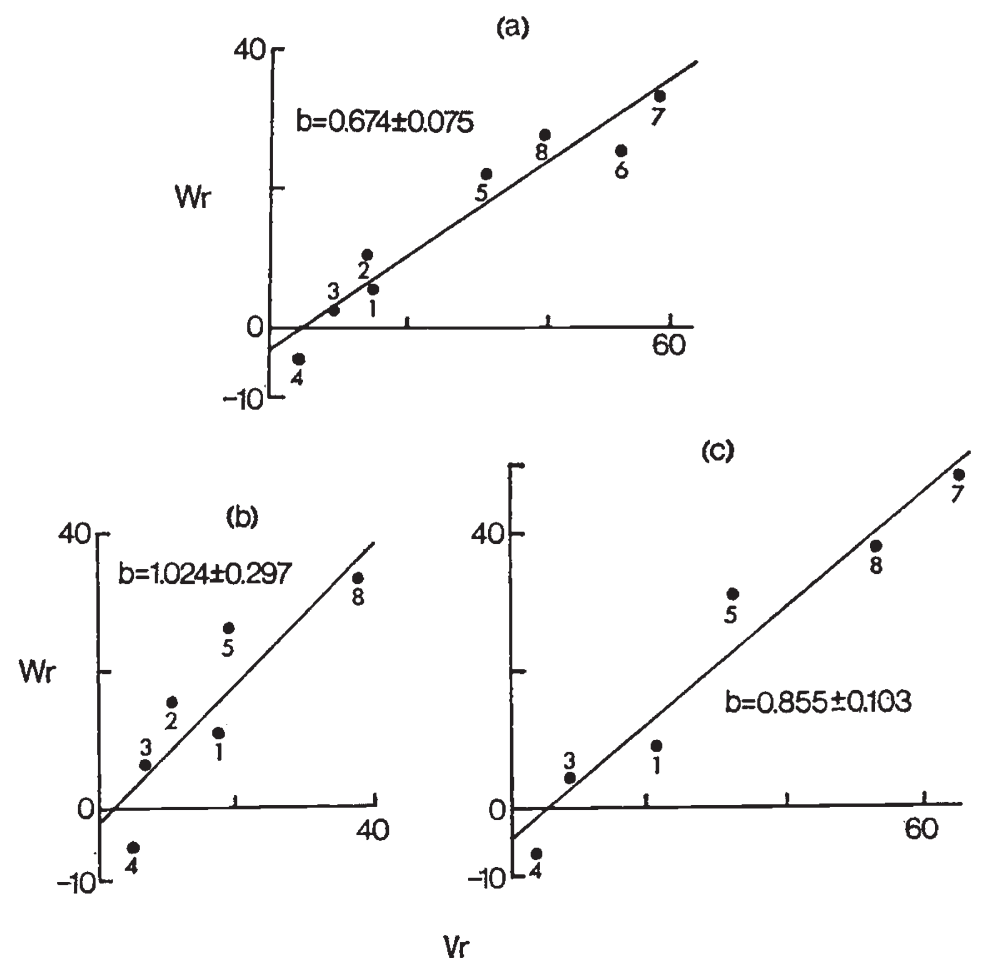

FIG. 5.-The regressions of $W r$ on $V r$ for plant height in 1971 ; (a) all eight parents, $(b)$ parents 6 and 7 omitted, (c) parents 2 and 6 omitted.

1972 data: Array differences in the magnitude of $W r+V r$ were highly significant $(\mathrm{P}=<0.001)$. Array differences were also significant $(\mathbf{P}=$ $0.025-0.05)$ for $W r-V r$ values suggesting non-additive genetic variance of the epistatic type. The joint regression coefficient of $W r$ on $\operatorname{Vr}(b=$ $0.577 \pm 0.058)$ differed significantly $(P=<0.001)$ from both zero and one. The difference in regression coefficients of the blocks was not significant $(P=0.50-0.75)$.

The departure from the expected rectilinearity, resulting possibly from complementary gene interaction, was detectable also from the graph given in fig. $6 a$. The $V r$ values were larger than $W r$ for all arrays. However, relatively large differences between $V r$ and $W r$ were evident for parental arrays $5,6,7$ and 8 , causing the slope of the regression line to drop significantly $(\mathbf{P}=0.01-0.001)$ below the expected value of one. In an attempt to determine if any single parent was responsible for most of the disturbances 
in the graph, each parent was omitted in turn and the remaining $7 \times 7$ diallel set was reanalysed. The omission of no single parent was found to be effective in improving the relationship between $W r$ and $V r$. However, when parents 7 and 8, corresponding to the two largest array variances (and also the largest differences between $V r$ and $W r$ ) were removed, the $6 \times 6$ diallel set was reduced to a rectilinear $W r$ and $V r$ graph with unit linear slope (fig. $6 b$ ).
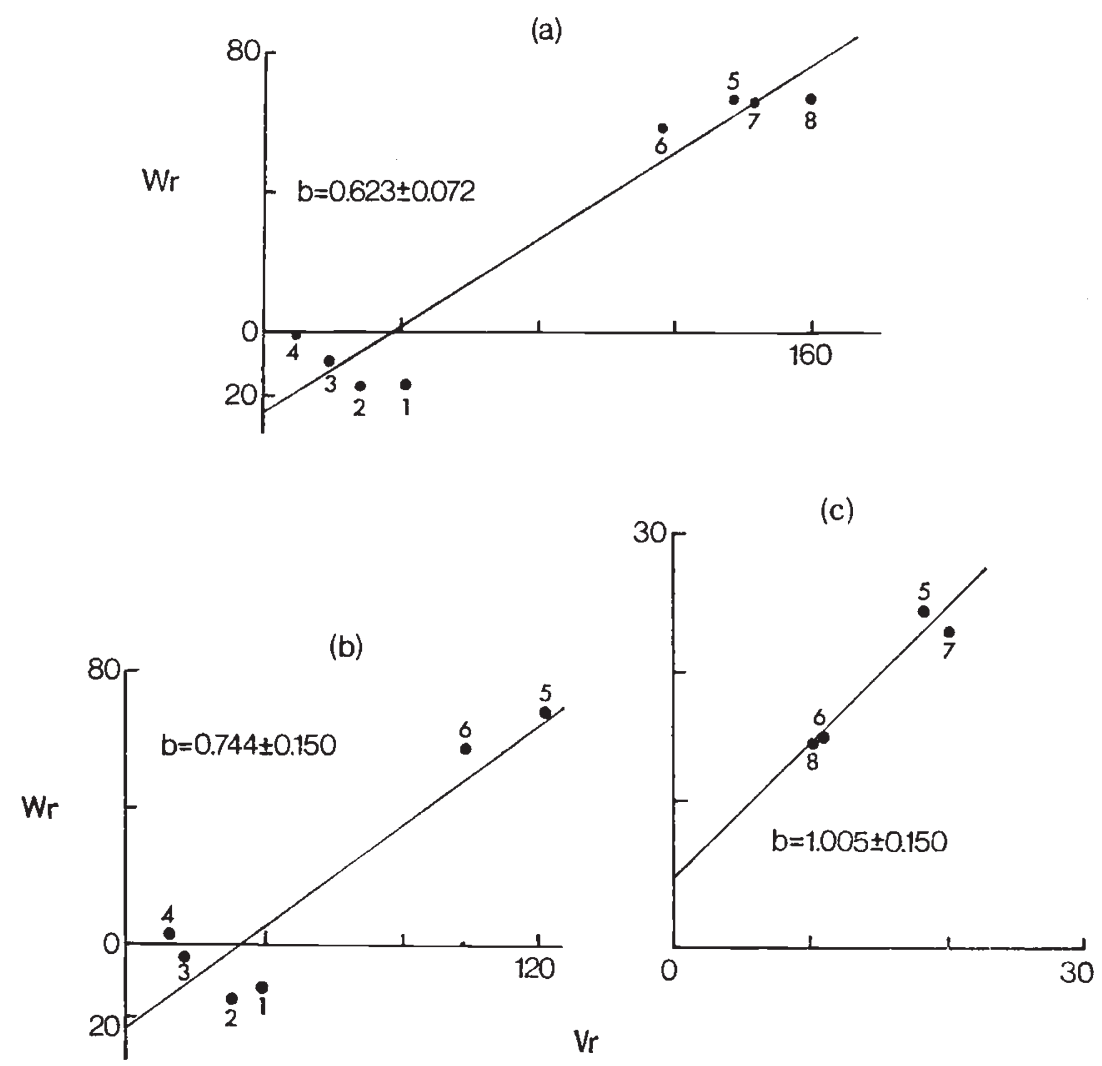

FIG. 6.-The regressions of $W r$ on $V r$ for plant height in 1972; (a) all eight parents, $(b)$ parents 7 and 8 omitted, (c) group A omitted.

The diallel graph for subdiallel set $A$ was unsatisfactory. Unlike in 1971, however, the graph of the subdiallel $B$ was satisfactory under the assumption of additive-dominance mode of gene action (6c). It is of interest to note that the disturbing effects of parents 6 and 7 found in the complete $8 \times 8$ diallel were not present in the subdiallel $B$, suggesting, therefore, that these parents were epistatic only in specific combinations with isogenic Atlas genotypes.

\section{(iv) Kernel weight}

An analysis of variance and a joint regression analysis showed that in neither year could the entire diallel cross data be interpreted on the basis 
of additive and dominance action of independently distributed genes (fig. $7 a$ and $b$ ). Removal of one, two or three parents at a time was ineffective in eliminating the causes of disturbance. In 1971 the inadequacy could be attributed to the isogenic parents since only when all of them were excluded, was the expected graph with unit slope attained (fig. 7c). However, the distribution of the array points in the graph was suggestive of a
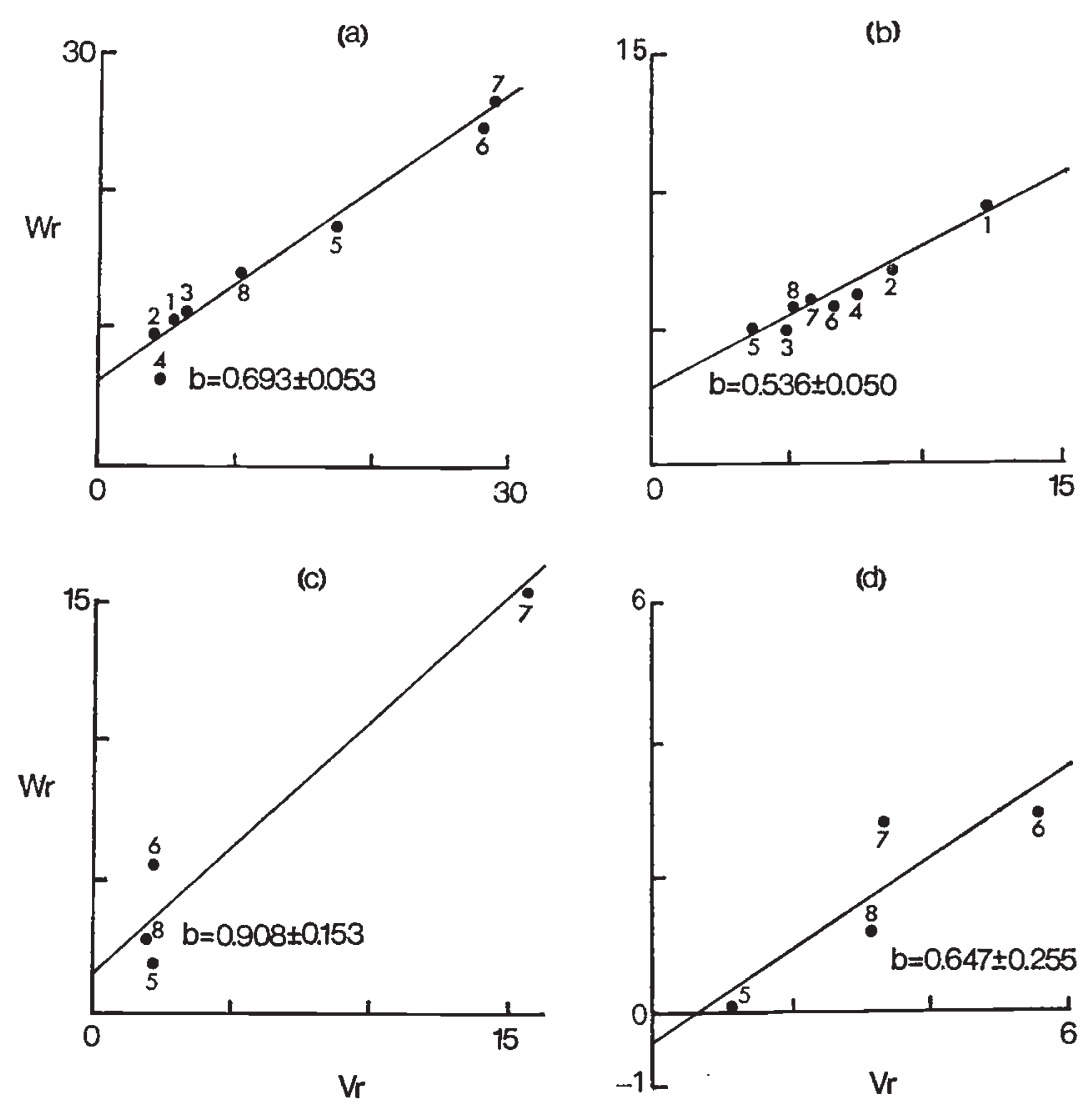

FIG. 7.-The regressions of $W r$ on $V r$ for kernel weight; (a) all eight parents in 1971, (b) all eight parents in 1972, (c) group A omitted in 1971, (d) group A omitted in 1972.

duplicate type of gene interaction. The estimates of $V r$ and $W r$ from the 1972 data of subdiallel $B$ failed to produce a satisfactory regression graph (fig. $7 d$ ), possibly due to disturbances caused jointly by epistasis and nonrandom gene distribution among a very small number of parents. The data from subdiallel $A$ were unsatisfactory for graphical analysis in both seasons.

\section{(v) Grain yield}

An analysis of variance and a joint regression analysis of $\mathrm{Vr}$ and $\mathrm{Wr}$ calculated from the data of both 1971 and 1972 indicated failures of one or more assumptions (fig. $8 a$ and $b$ ). Systematic eliminations of one, two or three parents at a time failed to remove the causes of failure. Removal of 
any three of the four isogenic lines and reanalyses of the reduced $5 \times 5$ diallel sets showed no difference in the relations of $\mathrm{Vr}$ and $\mathrm{Wr}$. The results suggest that, for grain yield, the contribution of the chromosome segments marked by the $A / a$ and $B / b$ loci to the total genetic diversity among the eight parents are relatively small. When the data from subdiallel $B$ were reanalysed, considerable improvement in the graphical relationship between $W r$ and $V r$ was observed in both seasons (fig. $8 c$ and $d$ ). Thus, the presence
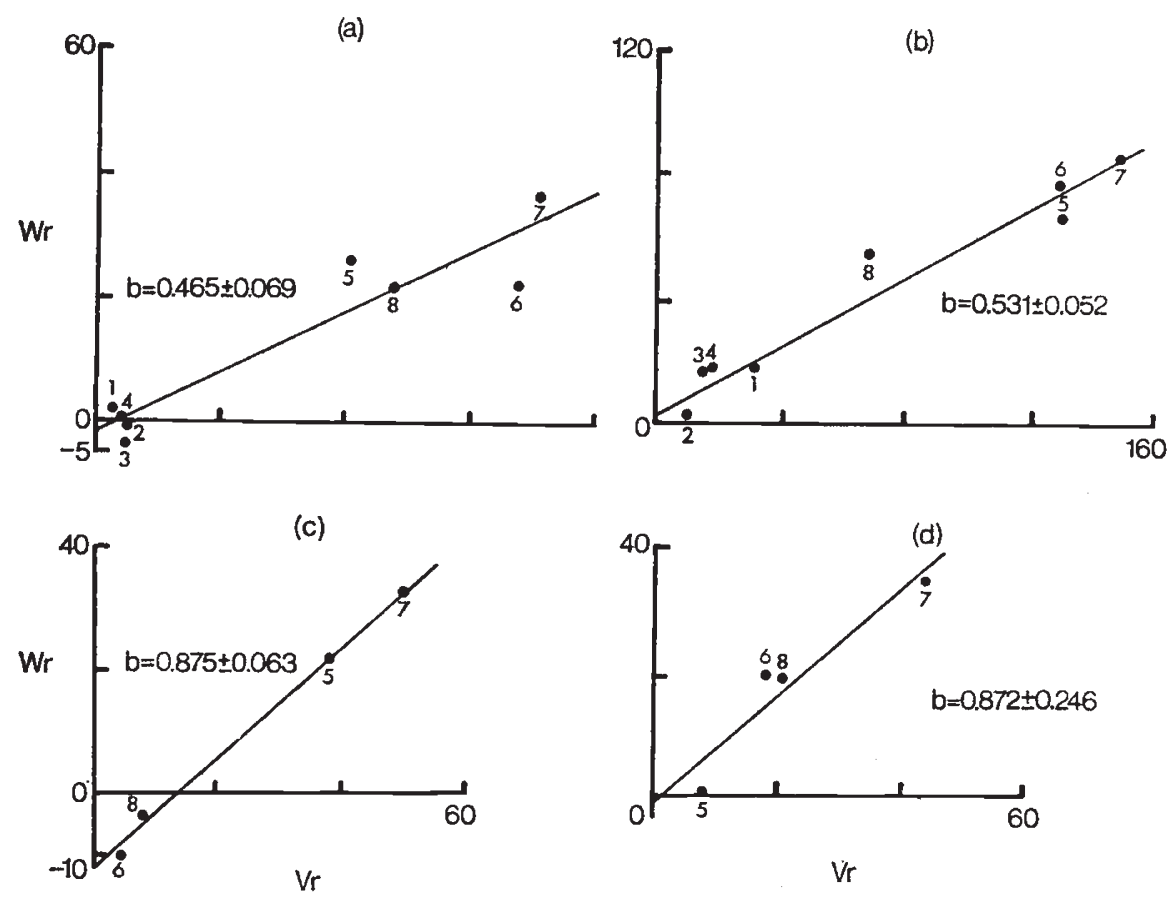

FIG. 8.- The regressions of $W r$ on $V r$ for grain yield; $(a)$ all eight parents in 1971, $(b)$ all eight parents in 1972, (c) group A omitted in 1971, (d) group A omitted in 1972.

of any one of the isogenic lines caused sufficient upsets in the $W r / V r$ relationship to invalidate a genetic interpretation of the data on the basis of the simplified model.

\section{Discussion}

The genetical implications of the graphical analysis of $W r$ and $V r$ statistics have been discussed by Jinks and Hayman in a series of papers since 1953, and more recently by Mather and Jinks (1971). The essential points to be gained from the analysis are $(a)$ the average dominance from the distance between the origin and $W r$-intercept of the regression line, (b) the relative proportion of dominant and recessive genes in the parents from the distribution of their respective array points along the regression line of unit slope, and $(c)$ a measure of genetical diversity among the parents from the distances between array points. It is well known that the above 
interpretations of the $W r / V r$ graph are possible only when a simple additivedominance model of gene action provides an adequate description of the data. In the presence of non-allelic interactions, the most useful information to be gained is about the existence of such interactions, since the graph itself, being sensitive to interactions, often permits their detection. Mather (1967) and Coughtrey and Mather (1970) have examined the consequences of correlated gene distribution and various non-allelic gene interactions with particular reference to the loss of the rectilinear relationship between $W r$ and $V r$ in the presence of dominance. It has been demonstrated that gene dispersion and association cause the $W r / V r$ graph to deviate from a straight line of unit slope in characteristic ways, which have a superficial similarity to the effects of complementary and duplicate interactions, respectively. Thus it becomes difficult to discriminate between these two possible phenomena affecting rectilinearity of the $W r / V r$ relation. Furthermore, the effects of duplicate interaction and gene association can be quite small causing no detectable departure from the expected linear regression of unit slope. Hence a significant regression $(\beta \neq 0)$ and no deviation from the slope of one may lead to an inaccurate conclusion regarding the mode of inheritance of a metrical character under investigation. The inherent insensitivity of the $\mathrm{F}_{1} W r / V r$ graph to the duplicate type of non-allelic interaction was demonstrated by Jinks. For flowering time in Nicotiana rustica, the diallel analysis of parental and $F_{1}$ means showed no detectable departure from the rectilinear relationship between $W r$ and $\operatorname{Vr}$ (Jinks, 1954). Later, a fuller analysis extending over the $F_{2}$ and backcross generations revealed that duplicate gene interactions played a significant role in the inheritance of flowering time (Jinks, 1956). The results of the present investigations involving only the $F_{1}$ and parental means must be considered in terms these limitations.

Days to maturity was the only character for which the complete $8 \times 8$ diallel population appeared to fulfil all the assumptions underlying diallel analysis. For the four other characters, days to heading, plant height, kernel weight and grain yield, a simple genetic basis of quantitative variation was found to be inadequate. When such discrepancies occurred, plant breeders and geneticists have routinely manipulated their data to salvage as much information as possible by eliminating those arrays which caused most of the discrepancies. If non-allelic interactions are the sole causes of disturbance, removal of one or two parents showing such interaction is often enough to make the remaining diallel data conform to an additivedominance model uncomplicated by epistasis and uncorrelated gene distribution.

For days to heading and plant height the complete $8 \times 8$ diallel data showed heterogeneity of $W r-V r$ and a significant departure of the $W r / V r$ graph from the unit linear slope. Omission of one or two supposedly epistatic parents resulted in the restoration of the rectilinear relation between $W r$ and $V r$ with unit slope. The parents omitted in each case were not necessarily the ones corresponding to the maximum variances or highest specific combining ability (estimates of the latter are not reported in this paper). The epistatic parents apparently did not follow the usual pattern of non-allelic interactions to be found in the literature on the application of diallel technique in genetical assays.

In the analysis of days to heading, at least one of the parents removed 
to produce a satisfactory $W r / V r$ graph belonged to group A. Similar desirable results were not obtained when othe members of the isogenic group were eliminated in turn. On the other hand, when only one of the isogenic parents was considered along with the varietal subset and the $5 \times 5$ diallel data were reanalysed, conspicuous differences were found in the slope of the regression line as well as in the degree of average dominance for all characters except grain yield. The genetical differences among the isogenic parents are ascribable to the allelic combinations at the marker loci $A / a$ and $B / b$ or at the most to the short chromosome segments marked by these genes in an otherwise isogenic background. The characters of concern here were quantitative in nature and presumably controlled by a large number of polygenes. The differential consequences on the $\mathrm{Wr} / \mathrm{Vr}$ graph of adding a different isogenic line to the $4 \times 4$ varietal diallel, or alternatively, of removing a different isogenic line from the complete $8 \times 8$ diallel table are inconsistent with the classical concept of small and similar effects of polygenes. The role of the $A / a$ and $B / b$ segments in the inheritance of days to heading, days to maturity and plant height was perhaps comparable to what were described by Mather and Jinks (1971) as the genes of intermediate kind. It is possible that polygenes were responsible for a major part of the differences among the parents, yet an appreciable part of the difference was attributable to the $A / a$ and $B / b$ segments with rather large individual effects.

In a small diallel cross of the size employed in the present study $(8 \times 8$ and smaller) non-random distribution of genes among the parents is normally expected to cause serious disturbances on the $W r / V r$ graph. For three characters, days to heading, days to maturity and plant height, this was not the case. The fact that in most cases satisfactory graphs were obtained despite drastic reductions in the number of parents entering the diallel table, suggests that non-allelic interaction might have been the more common cause of disturbance in the $W r / V r$ graph than non-random distribution of genes among the parents. It is of course possible that at least in some cases, the omission of parental arrays had merely reduced the diallel data to fit a unit linear regression line through the $(V r, W r)$ points without any real genetic significance. Although such a possibility could not be discounted, it was impossible to identify such cases. In the present study, therefore, simple genetic explanations as envisaged in the diallel theory are sought whenever the expected rectilinearity between $W r$ and $V r$ appeared to have been achieved. Under favourable circumstances this technique provides a reliable qualitative assay of the genetic relationships among parents entering a diallel cross. In the analysis of final plant height data in an eight-parent diallel cross in Nicotiana rustica, Jinks (1954) detected three parents which showed significant non-allelic interactions of a complementary type. After omitting these three parental arrays from the diallel table, he obtained the $W r / V r$ graph which did not differ significantly from the theoretical slope of one. Further tests involving the means of the $\mathrm{F}_{2}$ and backcross generations confirmed the presence of significant nonallelic interactions in crosses which included those parental lines (Jinks, 1956).

On the basis of the position of the regression line of unit slope it would appear that the average degree of dominance of the genetic systems in control of the three characters were quite similar, showing either complete 
dominance or slight overdominance. The estimates of correlation coefficients between the parental measurement $\left(Y_{r}\right)$ and the order of dominance $(W r+V r)$ were positive (not reported in this paper) in all these cases implying that dominance was active in the direction of early heading and maturity and reduced plant height. With the exception of heading time in 1972, most of the dominant alleles seemed to be present in the isogenic lines of Atlas. When these isogenic lines were eliminated altogether from the diallel table, the regression graph of the four parents in group B were consistent in showing partial dominance of the genes for earliness in heading and maturity and plant height. It seems, therefore, that the genetic systems controlling these traits did not differ substantially in average dominance and the distribution of dominant alleles in the non-epistatic parents. For kernel size and grain yield, however, no significant improvement of the $W r / V r$ graph was achieved by the application of the array elimination technique. The results suggest that a model more complex than one or two epistatic parents superimposed on an additive-dominance scheme of gene action must be invoked to describe the genetic basis of parental difference for these two characters.

A notable feature of the $W r / V r$ graph for days to heading was the complete reversal of the positions of the parents along the regression line in the two crop seasons. Not only were the disturbing parents different in the two years ( 1 and 7 in 1971 and 4 and 5 in 1972) but also when a satisfactory graph was obtained after their removal, the parents with most of the dominant alleles were from group B in 1971 and from group A in 1972. The results indicate a seasonal difference in epistatic effects of the parental genotypes as well as in dominance relationships among the non-epistatic parents. Although a reversal in the position of array points may be interpreted as different loci being active in different seasons, a more likely explanation would be the reversal of dominance effects at loci that control days to heading in both seasons.

The two epistatic parents causing upsets in the $W r / V r$ graph for plant heights were from group B in each year, and one (parent 7) was common in both seasons. Although there was a change in the preponderance of dominant and recessive alleles in the non-epistatic parents in the two seasons, the change was much less drastic than for heading time. The array points corresponding to the isogenic lines were closer to the origin in both years than were the points for the parents in group B. However, whereas in 1972 the parents could be classified into two distinct groups on the basis of their relative positions in the graph, the distribution of the array points were more or less continuous in 1971. In fact, some of the isogenic lines (e.g. 1 and 2) appeared closer to the largely different genotype, Bonanza (parent 5), than to their own isogenic relatives.

Barring a few exceptions such as days to maturity in 1972 (parent 4) and plant height in 1971 (parents 1 and 2), the expectation that the genotypic similarity of the isogenic lines would be reflected in the graphical representation of $\mathrm{Wr}$ and $\mathrm{Vr}$ statistics was borne out rather well. Contrary to the expectation, however, genetic differences among the four cultivars, Bonanza, Galt, Minnesota 5 and Gateway 63, appeared to be quite small and in some cases similar to the differences among the isogenic lines. In addition to the greater genetic affinity among the members of each group, the striking discontinuity in the distribution of the array points indicated 
substantial genetic differences between groups. Although a precise quantitative measure of genetic differences among the parents was not obtainable from the diallel graphs, if it is assumed that the genetic differences among the isogenic lines are limited to small chromosome segments marked by the $A / a$ and $B / b$ loci, then a comparative assessment of the differences among the four genotypes in group B becomes possible. Within the limits of their sampling errors, the estimates of $V r$ and $W r$, or the distance among the $(V r$, $W r$ ) points in the graph would provide a relative measure of genetic diversity among the common parents of the arrays represented by these points. As the closeness of the $(V r, W r)$ points of the isogenic parents is a reflection of their genetic similarity, the proximity of the array points of the four homozygous varieties must be interpreted as a comparable similarity of their genotypes. It should be mentioned here that the relative measures of genetic diversity available from the present graphs are applicable only to those genes which exhibit some degree of dominance. They provide no information on differences in respect to genes exhibiting no dominance. There is, however, no raison d'être for the supposition that the pattern of variation among the parents would be different if the genes showing no dominance could be taken into account. In an analysis of seed size data from a diallel cross involving closely related strains of lima beans, Allard (1956) found two distinct clusters of non-epistatic parents in the regression graph. He pointed out that in addition to the usual interpretation based on gene differences at several loci, such a discontinuity between the groups can be explained in terms of a 'single genetic unit with comparatively large influence'. Our unpublished work on simulated diallel populations also indicate that unequal genetic effects at a few diallelic loci can produce two distinctly separated groups of array points at opposite ends of the regression line within the limiting parabola. Whether the observed discontinuity between the two groups in the present experiment was due to the joint actions of many genes with small effects or a few (or single) genes with large effects is difficult to determine. It seems reasonable to conclude, however, that a larger number of genes were responsible for the difference between groups than within groups. With regard to the differences within groups $\mathrm{A}$ and $\mathrm{B}$, an interpretation in terms of a few genetic units is not only possible, but appears unavoidable.

Acknowledgment.-I am grateful to Professor R. W. Allard of the University of California at Davis and Professor J. L. Jinks of the University of Birmingham for critically reviewing the manuscript and suggesting many improvements.

\section{REFERENCES}

Allard, R. W. 1956. Biometrical approach to plant breeding. Proc. of Symp. on Genetics and Plant Breeding. Brookhaven Nat. Laboratory.

COUGHTREY, A., AND MATHER, K. 1970. Interaction and gene association and dispersion in diallel cross where gene frequencies are unequal. Heredity, 25, 79-88.

hayman, B. 1. 1954. The theory and analysis of diallel crosses. Genetics, 39, 789-809.

HAYMAN, B. 1. 1957. Interaction, heterosis and diallel crosses. Genetics, 42, 336-355.

hayman, B. I. 1963. Notes on diallel-cross theory. Statistical Genetics and Plant Breeding, NAS-NRG, 982, 571-578.

HILL, J. 1964. Effects of correlated gene distribution in the analysis of diallel crosses. Heredity, 19, 27-46. 
JINKs, J. L. 1954. The analysis of continuous variation in a diallel cross of Nicotiana rustica varieties. Genetics, 39, 767-788.

Jinks, J. L. 1956. The $\mathrm{F}_{2}$ and backcross generations from a set of diallel crosses. Heredity, 10, 1-30.

JINks, J. L., AND hayman, B. I. 1953. The analysis of diallel crosses. Maize Co-operation Newsletter, 27, 48-54.

Jornson, L. P. v. 1963. Application of the diallel-cross technique in plant breeding. Statistical Genetics and Plant Breeding, NAS-NRC, 982, 561-570.

MATHER, K. 1967. Complementary and duplicate gene interactions in biometrical genetics. Heredity, 22, 97-103.

MATHER, K., AND Jinks, J. L. 1971. Biometrical Genetics. Chapman and Hall, London.

NASSAR, R. F. 1965. Effects of correlated gene distribution due to sampling on the diallel analysis. Genetics, 52, 9-20.

QUALSET, c. o. 1968. Genotype-environment interaction for awn development in isogenic lines of barley. Theoret. Appl. Genet., 38, 355-360.

QUalset, C. O., SChaller, C. W., AND Williams, J. C. 1965. Performance of isogenic lines of barley as influenced by awn length, linkage blocks and environment. Crop Science, $5,489-494$.

SCHALlER, C. W., QUALSET, C. O., AND RUTGER, N. J. 1972. Isogenic analysis of the effects of the awn on productivity of barley. Crop Science, 12, 531-535. 